\title{
Study of the Recognition and Tracking Methods for Lane Lines Based on Image Edge Detections
}

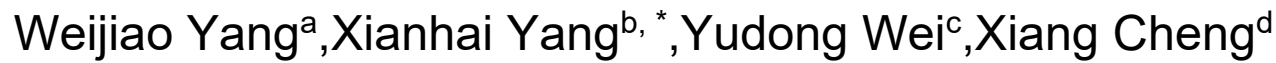

School of Mechanical Engineering, Shandong University of Technology, ZiBo 255000, China.

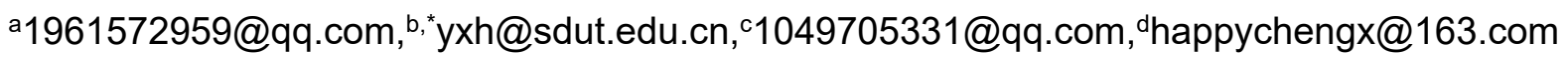

\begin{abstract}
In order to realize the real-time accurate recognition and tracking of lane lines for selfdriving cars, an improved Hough transform method for lane line recognition and fitting has been introduced based on the preprocessing of current frame lane line image. The extended Kalman filter is used to study the initial prediction and correction adjustments on the next frame lane line image. The relative distance between the vehicle and the lane line is to be obtained by the calculation of deviation rate. Then the detection and tacking of the lane line are realized. Experimental results prove that the introduced methods can effectively detect, recognize, and track the lane line for selfdriving cars with lower false detection rate and real-time detection abilities.
\end{abstract}

Keywords: Lane line detection, Constrained Hough transform, Extended Kalman filter, Lane tracking.

\section{Introduction}

In modern society, traffic has become an important part of everyday life. But accidents caused by traffic problems are continuously increasing. According to statistics, the annual number of deaths caused by traffic accidents reached more than 1.5 million[1]. Unmanned and computer assisted driving systems have been developed trying to ensure the safety of driving. The key technology and prerequisite for assisted driving or unmanned driving systems are accurate detection and tracking of lane lines. Therefore, how to take measures to ensure accurate and efficient recognition of lane tracking is of great significance. There are usually two types of features in lane detection, namely color features[2] and edges[3]. Many scholars have proposed a series of methods for lane detection and tracking. For example, Lin and Wang proposed a reconfigurable model[4-5] to identify lane markings. Gao et al. proposed a robust non-planar road lane detection algorithm, which improved the detection accuracy and computational speed[6]. Kim proposed a new improved lane line detection and tracking algorithm. The detected lane line targets were grouped by statistics, and the lane line segments were combined after grouping. Finally, the RANSAC algorithm was used for screening and fitting[7]. Guo et al. proposed a novel lane line detection algorithm that combines lane detections with vehicle identifications. The scientific research accurately extracts lane line parameters and has good resistance to vehicle disturbances[8]. When the road lane line is obstructed by obstacles, discontinuities such as discontinuities, or similar linear features of the lane line appear in the road, such as the linear shadow of a moving car, the boundary of a road etc will interfere with the recognition tracking effect, resulting in misjudgment of misjudgment. This article mainly aims at solving the above remaining problems, by constraining the interference factors, adding the early prediction and detections and correction module to the tracking recognitions, improving the accuracy of the detections and the fault tolerances of the tracking predictions.

\section{Composition of the Lane Line Detection and Tracking System}

The lane line detection and tracking system is based on the detection and recognition of image edges. The extended Kalman filter tracking algorithm is applied to realize the tracking process of the lane line. The entire method flow is as follows. First, the image is read and the lane line on the image edge is extracted. Then the decision is made whether the tracking module needs to be entered. If the image is the first frame or the last frame, the tracking is not needed and the lane line detection and fitting are directly conducted. Otherwise, it begins to enter the tracking module to track the lane line. If the tracking is successful, the state of the next frame is predicted according to the current frame state. When the prediction is found to be not accurate enough, fitting corrections are performed on 
the basis of the current frame. If the tracking fails, the process returns to start again. The flowchart is shown in Fig. 1.

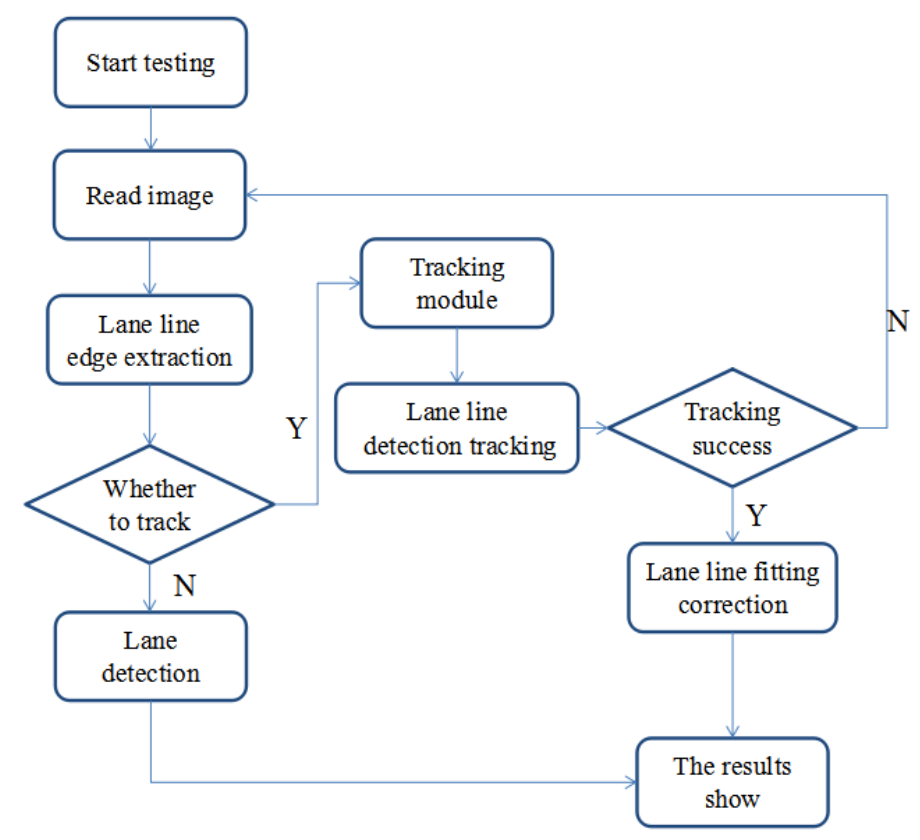

Fig. 1Lane line detection and tracking flowchart

The characteristics of road lane lines are studied and the lane line detection and tracking are divided into three modules, namely, (1) preprocessing module of lane line information, (2) lane line detection and identification module, (3)lane line tracking correction module. The three modules are explained as follows.

(1) The lane line preprocessing module simplifies the collected images, reduces its computational data and eliminates some of the interference factors. This module forms a solid basis for the following two modules.

(2) The lane line detection and recognition module will use the Hough transform with a constraint to fit the lane line edges obtained by preprocessing the road image to realize the purpose of recognizing the lane line from the road image.

(3) The lane line of the current frame will be identified. The tracking module will be used to predict and track the lane line. The position of the next lane line will be predicted by the state estimation method. When there is an inaccurate prediction or an improper fitting, the purpose of correct detection and fitting is to be realized by correcting the errors of the lane line predictions.

\section{Lane Line Detection and Tracking Methods}

\subsection{Lane Line Preprocessing Module.}

For complex road conditions, in order to reduce the interference factors in the recognition and detection processes and reduce the amount of data calculations, the road images are preprocessed. The processes are mainly divided into the following four steps as shown in Fig. 2.

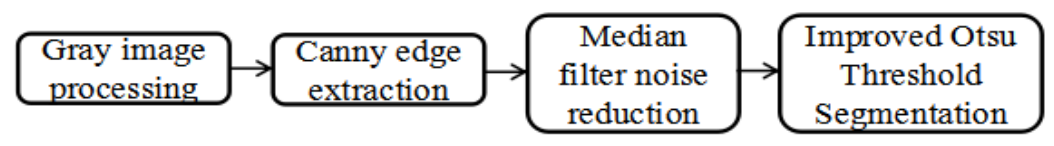

Fig. 2 Lane line preprocessing

(a) Gray-scale processing: the collected color image is converted to the gray-scale image to greatly reduce the amount of calculation. 
(b) Canny edge detection: edges that may be present in the image are extracted to prepare for the next step of fitting.

(c) Mean filter denoising: the filter is used to denoise the image to reduce the influence of noise on the detection since there is a large amount of noise in the processed image.

(d) Improved Otsu binarization process: the edge recognition is further enhanced by improving the Otsu binarization process to reduce the computational complexity of the edge detections. Then, the preprocessing of lane line detection and recognition is conducted. The experimental results are shown in Fig. 3.

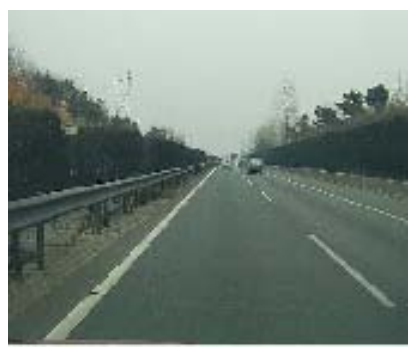

(a)Original image

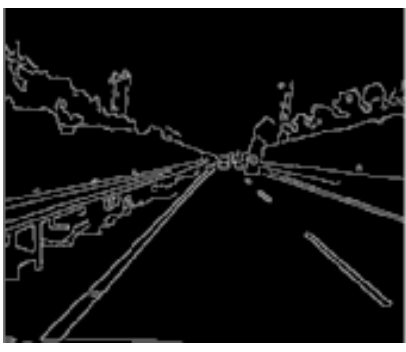

(c) Mean filter

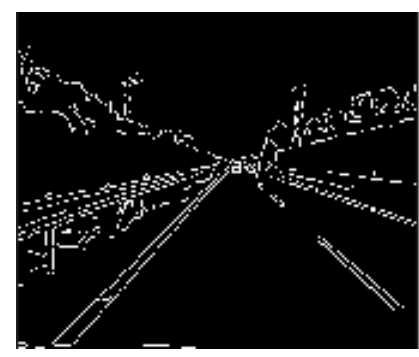

(b) Canny edge detection

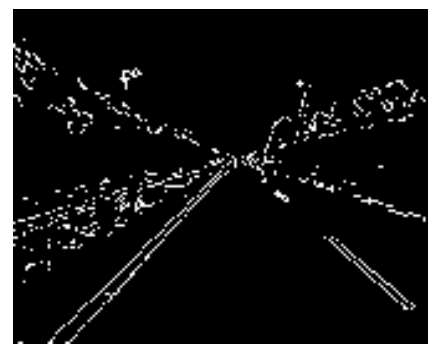

(d) Binary map

Fig. 3 Lane line preprocessing experiments

\subsection{Lane Line Detection and Identification Module.}

After the preprocessed edge feature image is fitted with a constrained Hough transform recognition. The algorithm detection and fitting process is as follows.

(1) The lane line edge feature of the road image is obtained by preprocessing.

(2) The parametric polar coordinates $\rho-\theta$ is established. The Hough transform of the image is calculated. The target point $(\mathrm{x}, \mathrm{y})$ on the image that needs to be transformed is searched using $\rho=\mathrm{x} \cos \theta+\mathrm{y} \sin \theta$ calculation parameters. The target is accumulated until all the conversion is completed to obtain a new Hough transform matrix[9]. Constraints are introduced first. The number of lines existing in the image is limited to $s<3$ and the slope angle difference must not be less than $6^{\circ}$ and $\left|k_{1}-k_{2}\right|>6$. Then, according to the visual images, the lane line almost shows in the lateral direction and the slope between $(-0.3 \leq \mathrm{k} \leq 0.3)$ can be deleted. The maximum value in the $\rho-\theta$ parametric space is identified on the premise of constraint conditions to obtain the corresponding parameters of the detection line.

(3) The Hough transform parameter coordinate is used to obtain the polar coordinate parameters $\rho-\theta$ of the detected line.

The lane image recognition and fitting are applied to the road image by using a constrained Hough transform. The experimental results are shown in Fig. 4. 

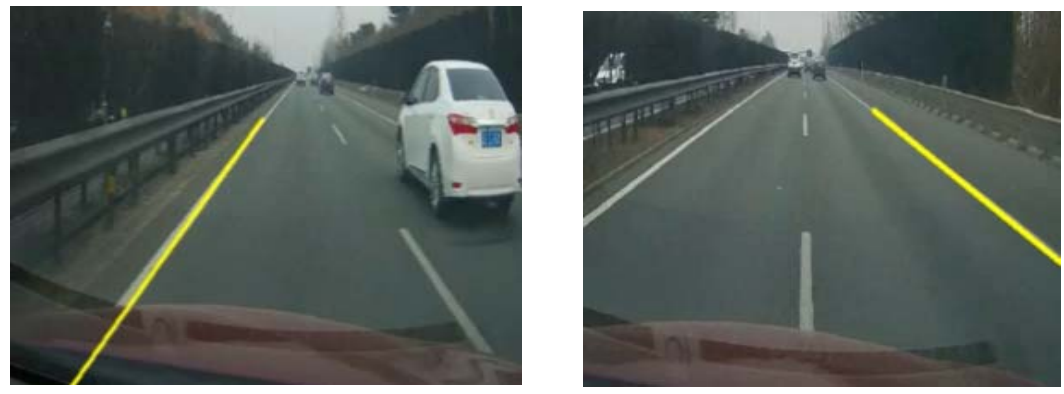

Fig. 4 Lane line recognition and fitting

\subsection{Lane Line Tracking Correction Module.}

For the moving and relatively moving targets, tracking detection must be performed during detection to realize the purpose of detection. The lane line tracking of the image is mainly based on the detection of the lane line result in the existing image. Then, the possible appearance position of the lane line in the road image sequence is predicted and estimated to determine the position of the lane line in the next frame. The lane line model parameters are evaluated to complete the lane line detection and tracking. The process of lane line tracking is shown in Fig. 5.

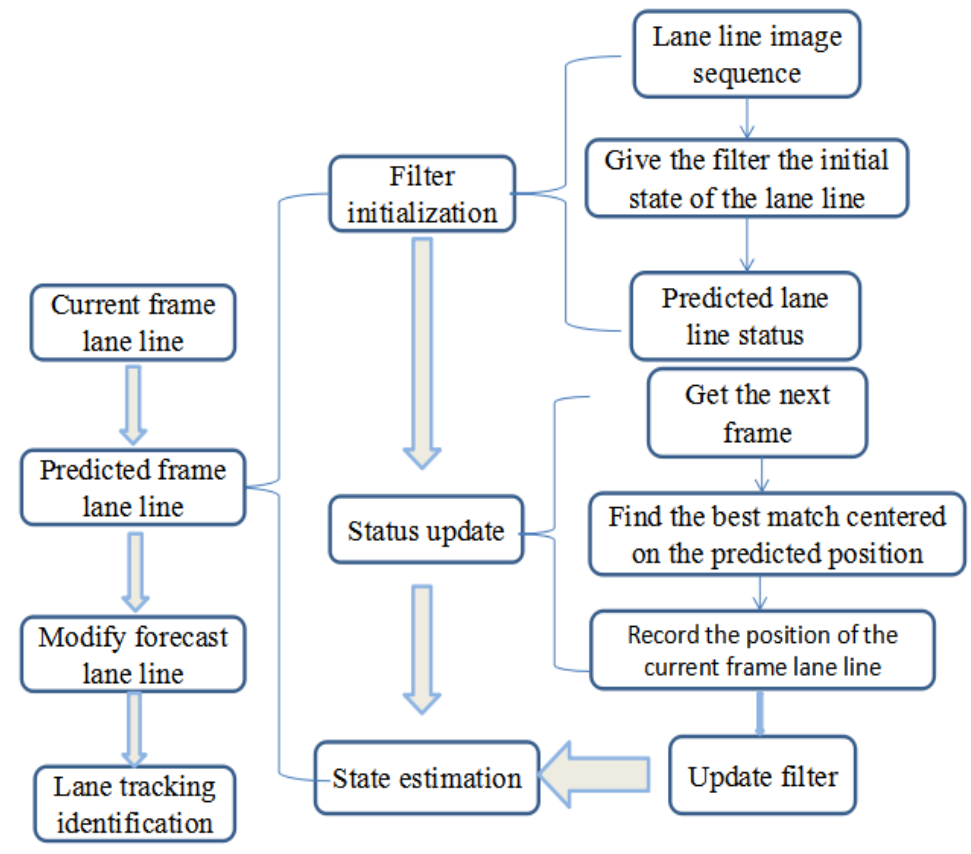

Fig. 5 Lane line tracking module flowchart

The lane line tracking correction method is mainly based on the extended Kalman filter (EKF) tracking prediction. The central idea is to use the Taylor expanded formula. The nonlinear equations are linearized. The slope of the lane line detected by the current frame is adjusted with the slope of the predetermined lane line. When the difference between the detection slope $k_{a}$ and the initial prediction slope $k_{b}$ is greater than $6^{\circ},\left(k_{a}+k_{b}\right) / 2$ is used as the prediction slope. Then, the detection and recognition are performed on the basis of prediction when next frame is detected.

The system model is assumed to have a nonlinear relationship.

$$
\begin{aligned}
& x(k)=f[x(k-1), w(k-1)] \\
& z(k)=h[x(k), v(k)]
\end{aligned}
$$

where, $x(k)$ is the state to be estimated by the system, $w(k-1)$ is the system process noise, $z(k)$ is the system measurement, $v(k)$ is the system test noise, and $f($.$) and h($.$) are the nonlinear process$ equations and measurement equations of the system, respectively[10]. 
The states of the process equation $f($.$) and the measurement equation h($.$) of the nonlinear system$ need to be estimated. The first-order Taylor series expansion equation of the nonlinear function $x(k)$ at $w(k-1)=0$ And $x(k-1)=\hat{x}(k-1 \mid k-1)$ is shown in equation (1).

$$
x(k)=\left.\frac{\partial f}{\partial x}\right|_{\hat{x}(k-1 \mid k-1)} x(k-1)\left[f(\hat{x}(k-1 \mid k-1), 0)-\left.\frac{\partial f}{\partial x}\right|_{\hat{x}(k-1 \mid k-1)} \hat{x}(k-1 \mid k-1)\right]+\left.\frac{\partial f}{\partial w}\right|_{\hat{x}(k-1 \mid k-1)} w(k-1)
$$

Assume,

$$
\left\{\begin{array}{l}
F(k)=\left.\frac{\partial f}{\partial x}\right|_{\hat{x}(k-1 \mid k-1)} \\
L(k)=\left.\frac{\partial f}{\partial w}\right|_{\hat{x}(k-1 \mid k-1)}
\end{array}\right.
$$

Equation (3) is obtained combining equations (1) and (2).

$$
x(k)=F(k) x(k-1)+[f(\hat{x}(k-1 \mid k-1), 0)-F(x) \hat{x}(k-1 \mid k-1)]+L(k) w(k-1)
$$

The more Taylor series are expanded, the higher the accuracy will be. The linear relationship $F(x)$ of state transition is given by the above euqations. The Kalman filter can be used to realize the state estimation. However, after the Taylor series expansion, the variance of the process noise has also changed from the original $Q(k)$ to $L(k) Q(k) L^{T}(k)$ when $v(k)=0$ and $x(k)=\hat{x}(k \mid k-1)$.

$$
y(k)=\left.\frac{\partial h}{\partial x}\right|_{x=\hat{x}(k \mid k-1)} x(k)+\left[h(\hat{x}(k \mid k-1), 0)-\left.\frac{\partial h}{\partial x}\right|_{x=\hat{x}(k \mid k-1)} \hat{x}(k \mid k-1)\right]+\left.\frac{\partial h}{\partial v}\right|_{x=\hat{x}(k \mid k-1)} v(k)
$$

$$
\left\{\begin{array}{l}
H(k)=\left.\frac{\partial h}{\partial x}\right|_{x=\hat{x}(k \mid k-1)} \\
M(k)=\left.\frac{\partial h}{\partial v}\right|_{x=\hat{x}(k \mid k-1)}
\end{array}\right.
$$

Equation (6) is obtained by combining equations (4) and (5).

$$
y(k)=H(k) x(k)+\left[h(\hat{x}(k \mid k-1), 0)-\left.\frac{\partial h}{\partial x}\right|_{x=\hat{x}(k \mid k-1)} \hat{x}(k \mid k-1)\right]+M(k) v(k)
$$

The lane line detection recognition and tracking method mainly detects the fitted lane line through the current frame, and compares the extended Kalman filter tracking estimation with the currently fitted lane line to determine the position and fitting of the next lane line. The lane line of the current frame is set to yellow, and the lane line fitting color of the next predicted frame is to be light green. It is convenient to distinguish the slope of the current frame to adjust the slope of the next frame. It is more convenient to see the difference between the current frame and the predicted frame of lane line fitting. The calculation of the deviation rate is performed by the distance between the center of the driving recorder image and the left and right lane lines on both sides of the plane projection.

\section{Experimental Results of Lane Line Detection and Tracking}

The video samples in the experiments are from video recorder on high-speed road sections from Qingdao to Rizhao. It detects and tracks the lane line through the lane detection and the extended Kalman filter tracking method. This article is based on the hardware of the Intel®Core TQMi76700CPU@3.40 GHz processor and 64-bit operating system. The simulation is performed by MATLAB2016a software. The experimental results are shown in Fig. 6. If the vehicle deviates from the left lane line, it will be positive. If the vehicle hits the left lane line, it will deviate by $+100 \%$. If 
the vehicle deviates from the right lane, it will be negative. If the vehicle hits the right lane line, it will deviate by $-100 \%$, as shown in Table 1 .

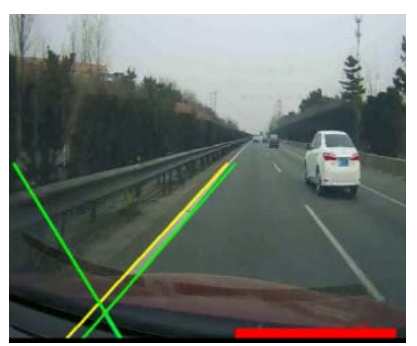

(a)

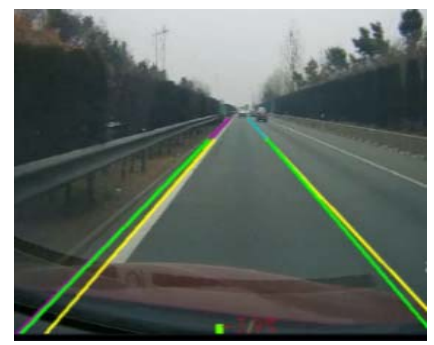

(e)

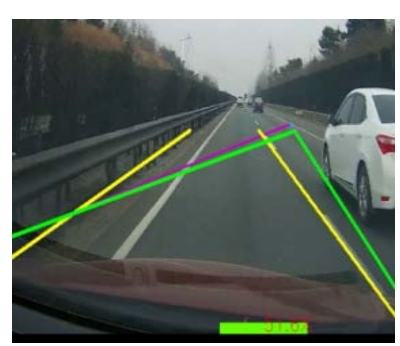

(b)

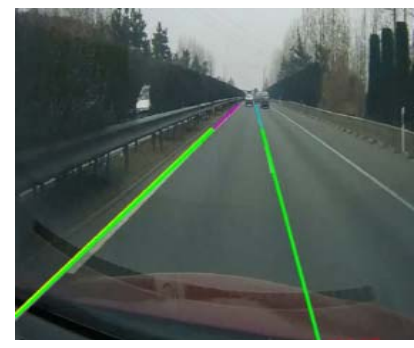

(f)

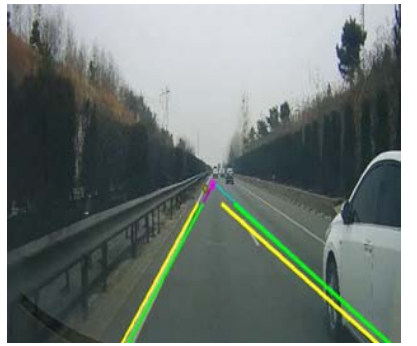

(c)

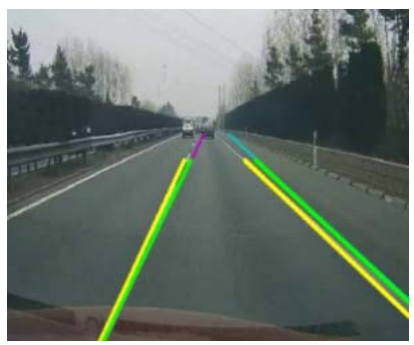

(g)

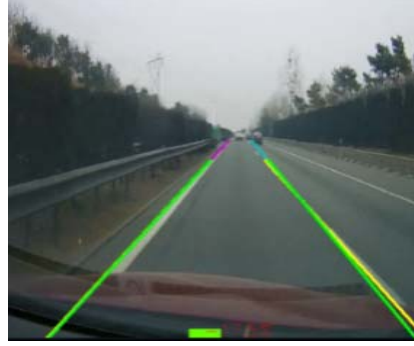

(d)

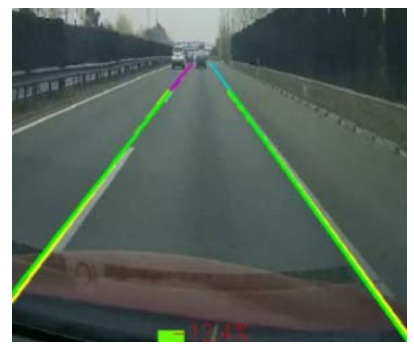

(h)

Fig. 6 Lane line detection and tracking experiments

Table 1.Lane line deviation rate parameters

\begin{tabular}{ccc}
\hline Image & Number of frames & Lane departure percentage \\
\hline (a) & 35 & $+100 \%$ \\
(b) & 60 & $+51.6 \%$ \\
(c) & 110 & $-76.7 \%$ \\
(d) & 135 & $-17.5 \%$ \\
(e) & 185 & $-3.9 \%$ \\
(f) & 210 & $-100 \%$ \\
(g) & 235 & $-100 \%$ \\
(h) & 260 & $-12.4 \%$ \\
\hline
\end{tabular}

False detection as shown in Fig. 6(a) occurs since the vehicle is not adjusted well at the beginning. The tracking result is unstable after the start of the test and the correction and fitting is not in time as shown in Fig. 6(b). The situations, through the subsequent debugging detection and correction tracking, gradually become normal as shown in Fig. 6(c) to (h).

As shown in Fig. 6 and Table 1, when the on-board camera detects that the position of the vehicle on the road collides with the left lane line, the system displays a deviating rate of $+100 \%$. When the vehicle collides with the right lane line, the system shows that the deviation rate is $-100 \%$. The deviation rate is determined based on the vehicle positions from the lane lines on both sides judged by the onboard camera. When the vehicle deviates the road or changes lanes, the positive and negative values and the magnitude of the deviation rate are displayed according to the direction and degree of deviation. When only a single lane line is detected, the deviation rate cannot be judged based on the distance between the left and right lane lines, and the deviation is not calculated. Only when the detected left and right lane lines is determined by the ratio of the difference between the two sides, the deviation rate shown in Table 1 is less than $100 \%$. It shows that the vehicle is inside the lane line and there is no deviation. The deviating rate of Fig. 6(f) and (g) in Table 1 reaches $100 \%$, which is caused by the lane changes of the vehicle. 


\section{Conclusion}

This paper mainly studies the detection and tracking methods of road lane lines for the self-driving vehicles. The lane line images of the current frame are detected and the Hough transform with constraints are used for recognition and fitting. The extended Kalman filter is applied to predict and identify the next frame to achieve the purpose of tracking. When there is a difference between the predicted lane line and the target one, it is adjusted and tracked by the slope fitting to the lane line of the current frame to realize the detection, recognition and tracking process of the lane line based on the images. This method can effectively perform the fitting recognition and tracking according to the trajectory of the front road lane lines. The false detection and false detection rate can be effectively reduced. This method is ineffective for the unclear lane lines conditions. Therefore, in the future, the unstructured road boundary conditions should be studied in depth.

\section{Acknowledgments}

Funds: National Natural Science Foundation of China (61573009);Natural Science Foundation of Shandong Province (ZR2014EEM046).

\section{References}

[1]. ZIYAN A H,AKHTAR S.Incidence and trend of road traffic injuries and related death sinKuwait:2000-2009[J].Injury,2012,43(12):2018-2022.

[2]. T.Y.Sun,S.J.Tsai,V.Chan. HSI color model based lane marking detection,in:IEEE on Intelligent Transportation Systems Conference,2003.pp.1168-1172.

[3]. Y.Wang, N.Dahnoun. A novel system for robust lane detection and tracking. Signal Process.92(2) (2012)319-334.

[4]. L.Lin,X.Wang,W.Yang,et.Discriminatively trained and-or graph models for object shape detection, IEEETrans.Pattern Anal.Mach,intell.37(5)(2015)959-972.

[5]. X.Wang,L.Lin,LHuang,S.Yan.Incorporating structural alternatives and sharing into hierarchy for multiclass object recoginition and detection,in:2013IEEE Conference on Computer Vision and Pattern Recognition (CVPR),pp.3334-3341.

[6]. J.Li, J.P.Chen, Y.Ke-chuan,C.S.Xu. Feature Recognition based Vision Navigation for Smart Vehic-les [J].Automotive Engineering,2015,37(05):587-592.

[7]. S.Z.Li. Design and implementation of lane line detection system based on deep learning[D].Harbin Institute of Technology,2016.

[8]. L.Guo, J.Q.Wang, K.Q.Li. Lane Line Identification with Vehicle Interference [J]. Automotive Engin-eering, 2007,(05):372-376+400.

[9]. R.J.Duan,W.Zhao,S.L,Huang,et.A Fast Algorithm for Straight Line Detection Based on Improved Hough Transform[J].Chinese Journal of Scientific Instrument,2010,31(12):27742780.

[10]. Z.Luo System State Estimation and Fault Detection Based on Kalman Filter[D]. Huazhong Univer-sity of Science and Technology,2013. 